\title{
Notch signaling as a therapeutic target for breast cancer treatment?
}

\author{
Jianxun Han, Michael J Hendzel and Joan Allalunis-Turner*
}

\begin{abstract}
Aberrant Notch signaling can induce mammary gland carcinoma in transgenic mice, and high expressions of Notch receptors and ligands have been linked to poor clinical outcomes in human patients with breast cancer. This suggests that inhibition of Notch signaling may be beneficial for breast cancer treatment. In this review, we critically evaluate the evidence that supports or challenges the hypothesis that inhibition of Notch signaling would be advantageous in breast cancer management. We find that there are many remaining uncertainties that must be addressed experimentally if we are to exploit inhibition of Notch signaling as a treatment approach in breast cancer. Nonetheless, Notch inhibition, in combination with other therapies, is a promising avenue for future management of breast cancer. Furthermore, since aberrant Notch 4 activity can induce mammary gland carcinoma in the absence of RBPjk, a better understanding of the components of RBPjk-independent oncogenic Notch signaling pathways and their contribution to Notch-induced tumorigenesis would facilitate the deployment of Notch inhibition strategies for effective treatment of breast cancer.
\end{abstract}

\section{Introduction}

Notch signaling is an evolutionarily conserved pathway that is essential for embryonic development, organogenesis, and tissue homeostasis. Aberrant Notch signaling is associated with several inherited developmental diseases and various types of cancer. The membraneanchored Notch receptor is cleaved into two fragments post-translationally and is assembled into the plasma membrane as a non-covalently linked heterodimer of the $\mathrm{N}$-terminal and C-terminal fragments. Upon ligand binding, the bipartite receptor undergoes at least two

*Correspondence: joan.turner@ualberta.ca

Department of Oncology, University of Alberta, Cross Cancer Institute,

11560 University Avenue, Edmonton, AB, Canada T6G 1 Z2 sequential cleavages - with the last one mediated by the $\gamma$-secretase complex - to release the active intracellular domain (NICD). NICD then translocates into the nucleus to form a trimeric core transactivation complex with the sequence-specific DNA-binding protein, CSL (CBF-1/ $\mathrm{Su}(\mathrm{H}) / \mathrm{Lag}-1)$, and Mastermind or Mastermind-like protein (MAML), which further recruits other transcription activators to activate the transcription of Notch target genes. The four mammalian Notch paralogs share similar structural motifs and the same activation processes and interact with the same CSL and MAML proteins (reviewed in [1]).

A possible link between Notch signaling and breast cancer was first noted when a hot spot for mouse mammary tumor virus (MMTV) insertional mutagenesis, Int3, was found to be Notch4 [2]. Sequence analysis of the host-viral junctions revealed that all of the tumorigenic MMTV insertions occurred between the negative regulatory region (NRR) and the transmembrane domain, which ultimately produce high int3/Notch4 intracellular domain (N4ICD) levels [2]. Expression of the intracellular domain of int3/Notch4 specifically in developing secretary mammary epithelium induced mammary carcinoma, confirming that aberrant int3/Notch4 signaling is tumorigenic [3]. Later, transgenic mice expressing constitutively active N1ICD or N3ICD in mammary epithelium were also shown to develop mammary gland carcinomas [4]. These observations, together with the report that high expression of Jagged1, a Notch ligand, or Notch1 (or both) which likely leads to elevated Notch signaling is associated with poor clinical outcomes [5], have stimulated intense interest in exploring Notch signaling as a therapeutic target for breast cancer treatment (reviewed in $[6,7])$. This review critically evaluates the evidence that supports or challenges the hypothesis that the inhibition of Notch signaling has therapeutic potential in the treatment of breast cancer.

\section{Is Notch signaling aberrantly activated in breast cancer?}

Unlike T-cell acute lymphoblastic leukemia (T-ALL), in which more than half of patients have an activating mutation in Notch1 [8], a chromosomal translocation 
involving Notch loci or an activating mutation has yet to be reported in breast cancer. In T-ALL, activating mutations are commonly found in the Notch 1 NRR that regulates NICD production and in the proline/glutamic acid/serine/threonine-rich (PEST) domain, which regulates NICD turnover. However, among 48 breast cancer samples, only one nonsense mutation in Notch2, which produced a truncated PEST domain and potentially enhanced Notch2 signaling, was found [9]. No mutations in the other three Notch receptors were observed. However, as will be discussed later, the functional consequence of Notch2 activation - suppressing or promoting breast tumorigenesis - remains to be investigated. The absence of mutations raises the critical question of how Notch signaling could be activated and pathological in breast cancer. Two possible explanations have been proposed: (a) higher expression of Notch receptors or ligands or both and (b) loss of the negative regulator, Numb.

Several studies reported that the expression of Notch receptor and ligand proteins is higher in breast cancer tissues than in normal breast epithelium [10-13]. Rizzo and colleagues [12] examined the expression of Notch1, Notch4, Jagged1, and Dll1 in 4 normal breast tissues, 5 hyperplasias of usual type (HUTs), 27 ductal carcinomas in situ (DCISs), 27 infiltrating ductal carcinomas (IDCs), and 14 infiltrating lobular carcinomas (ILCs) by immunohistochemistry. None of the normal breast tissues expressed high levels of Notch1 or Notch4. In contrast, $80 \%$ of HUTs, $67 \%$ of DCISs, $89 \%$ of IDCs, and $57 \%$ of ILCs expressed high levels of Notch1. High Notch4 levels were not detected in any HUT or DCIS samples but were present in $81 \%$ of IDCs and $93 \%$ of ILCs. The expression of Jagged1 and Dll1 was not examined in normal tissues, HUTs, or DCISs, but $78 \%$ of IDCs and $64 \%$ of ILCs expressed high Jagged1 levels. Similarly, another study reported higher expression level of multiple Notch receptors and ligands in IDC compared with normal breast tissues [13].

In a later study, Zardawi and colleagues [11] used tissue microarrays to examine Notch1 expression in 693 samples from 222 patients. Surprisingly, although the authors found a gradual increase in the percentage of high Notch1 expression cases from normal breast tissues (13.6\%) to grade 2 or 3 DCISs (57\% to 59\%), which is consistent with the notion that Notch1 signaling is activated in breast cancer and promotes tumor progression, the expression level of Notch in IDCs was lower than that in DCISs. More surprisingly, a recent study that examined 79 normal breast and 408 breast cancer samples, including 367 IDC and 29 ILC cases, found no difference in the expression of Notch 1 protein between breast cancer and normal breast tissues [14]. Therefore, whether expression of Notch receptors and ligands, especially Notch1 protein, is elevated in breast cancer tissues, or whether their expression level is increased during early stages of breast cancer development but declines during later stages, remains to be clarified.

Another proposed mechanism for aberrant Notch activation in breast cancer is the loss of a Notch-negative regulator, Numb. It is generally believed that Numb downregulates Notch signaling by promoting its polyubiquitination and lysosomal degradation or by regulating its endocytosis (or both), consequently reducing its availability on the cell surface, where it responds to ligand binding-induced activation [15]. Numb was found to be absent or at low levels in approximately $50 \%$ of breast cancer samples and this was due to enhanced proteasomal degradation [16]. Consistent with this, Stylianou and colleagues [17] detected N1ICD (the active form of Notch1) in 20 breast cancer samples from which Numb was absent. However, the correlation between Notch activation and loss of Numb needs to be examined in a larger group of patients as a recent study of 36 IDC cases failed to demonstrate a direct correlation [13].

It should be emphasized that, while both elevated expression of Notch receptors and ligands and loss of Numb suggest the possibility of aberrant Notch activation, they are not, per se, equivalent to enhanced Notch signaling, which needs to be verified by the presence of active NICD and upregulated expression of Notch target genes. To this end, Mittal and colleagues [13] reported that both active N1ICD and Hes1/5 protein could be detected in 27 out of 35 IDC samples but were present in only 1 of 13 normal breast tissues. N1ICD was detected in the nucleus and cytoplasm. Since active NICD in cultured breast cancer cells is always present in nuclei, the nature of cytoplasmic N1ICD in this study needs to be clarified further, as does the specificity of the primary antibody used. Furthermore, others have failed to find a difference in Hes1 expression when comparing normal breast tissues with breast cancer tissues [18]. This raises concerns as to whether Notch is aberrantly activated or whether Hes1 is even a Notch target gene in breast cancer tissues.

Even if Notch signaling is more active in breast cancer compared with normal breast tissue, it remains to be determined whether the activation is sufficient to drive breast oncogenesis. Also, it is not known whether breast tumorigenesis requires the cooperation of other signaling pathways with Notch. This might be expected given that several activating Notch mutant alleles commonly found in T-ALL cannot initiate oncogenesis alone, despite their ability to activate several Notch target genes [19].

\section{Notch and breast cancer progression}

Interest in exploring Notch signaling as a potential target for breast cancer treatment stems from early reports that 
high expression levels of Notch ligands or receptors or both, which potentially result in aberrant Notch activity, were correlated with poor clinical outcomes $[5,10]$. Reedijk and colleagues [5] reported that high levels of Jagged1, Notch1, or Notch3 mRNA were correlated with increased mortality in two independent cohorts of breast cancer samples; and the 5-year survival rates for the high- versus low-expression groups were $42 \%$ versus $65 \%$ for Jagged1, $49 \%$ versus $64 \%$ for Notch 1 , and $48 \%$ versus $61 \%$ for Notch3. Later, in an expanded study that included 887 patients with lymph node-negative breast cancer, the same group found that Jagged1 expression still had predictive value [20]. However, a subsequent study that analyzed two publicly available breast cancer gene expression profile datasets found that Notch1 mRNA levels were not of prognostic significance [11]. In addition, Notch1 protein levels were not of prognostic value in a cohort of 228 patients with breast cancer. Therefore, it remains to be determined whether aberrant Notch signaling is associated with breast cancer progression and clinical outcome and, if so, whether it is paralog-specific.

\section{Jagged 1 and bone metastasis}

A very recent publication provides an intriguing alternative explanation of how high Jagged1 expression could be associated with poor clinical outcome through mechanisms other than activating Notch signaling and promoting proliferation in breast cancer cells [21]. Sethi and colleagues [21] observed that the expression of Jagged1, but not that of Notch receptors, is higher in high-metastatic cell lines than in their low-metastatic counterparts and that it positively correlated with the incidence of bone metastasis in patients with breast cancer. Knocking down or overexpressing Jagged1 in breast cancer cell lines inhibited or enhanced their bone metastatic capacity, respectively, without affecting the growth of cultured cells or of primary mammary tumors. Further investigation revealed that this pro-metastasis function of tumor-derived Jagged1 was mediated by at least two mechanisms: (a) stimulating osteoblasts to release interleukin- 6 that, in turn, promotes the growth of cancer cells and (b) inducing severe osteolysis by promoting osteocalst differentiation, thus providing space for metastatic breast cancer cells to proliferate. The ability of MRK-003, a specific $\gamma$-secretase inhibitor (GSI), to delay the onset of bone metastasis suggests that blocking Notch activation in the bone marrow microenvironment might prevent or reduce bone metastasis in patients with breast cancer.

\section{Notch and subtypes of breast cancer}

Since breast cancer is composed of several subtypes associated with distinct gene expression profiles and different clinical outcomes [22], it is possible that aberrant Notch signaling is associated with the oncogenesis and progression of a particular breast cancer subtype. Using a cohort of 611 patients with breast cancer, Dickson and colleagues [23] first reported that high Jagged1 mRNA levels are associated with basal-like breast cancers: estrogen receptor (ER)-negative and CK5-positive. Interestingly, they found that there was no direct correlation between Jagged1 mRNA and protein levels and that high Jagged1 protein level was associated with ER-positive, rather than ER-negative, patients. Similarly, Lee and colleagues [9] suggested that high Notch1 mRNA levels are associated with basal type breast cancer, but two other studies found that high levels of Notch1 protein were associated with HER-2 and luminal types of breast cancer, respectively $[11,14]$. Since it is the protein, not the mRNA, levels of Notch receptors and ligands that dictate the activation status of Notch signaling, studies that examine protein levels should be more reliable. However, it should be noted that, in the study that suggested an association of Notch1 with HER-2 type, only 17 of 224 patients were classified as HER-2 [11]. Therefore, the association might not hold when a larger group of HER-2 type patients is examined.

On the other hand, studies with established breast cancer cell lines have suggested that higher Notch activity, as measured by an RBPjk-dependent reporter, was associated with HER-2-negative or ER/progesterone receptor (PR)/HER-2-triple-negative - commonly regarded as basal-like - breast cancer cell lines, although the difference between triple-negative MDA-MB-231 and ER/PR-positive MCF-7 was statistically significant in one study [12] but not in the other [24]. This is consistent with the reported high expression levels of Jagged 1 and selective cytotoxicity of Jagged1 knockdown in triplenegative breast cancer cell lines [25] and the selective cytotoxicity of Notch3 knockdown in HER-2-negative breast cancer cell lines [26]. However, in another study [21], Jagged1 knockdown did not affect the proliferation of MDA-MB-231 cells in vitro or as primary mammary tumors, although it did suppress bone metastasis. In addition, as will be described below, blocking Notch activation with specific GSIs is not selectively toxic to HER-2-negative or -triple-negative breast cancer cells in culture $[27,28]$. Therefore, these discrepancies need to be addressed to confirm the association of higher Notch activity with HER-2-negative or -triple-negative breast cancers.

\section{Functional differences among Notch paralogs in breast cancer}

Most studies, especially those examining the status of Notch receptors in clinical samples, have focused on the Notch1 paralog, although there is no evidence that other 
Notch paralogs play a less important role in breast cancer. It should be noted that the four Notch paralogs are thought to play different, even opposing, roles in breast cancer, a fact that complicates the design of therapeutic strategies to block Notch signaling $[10,29]$.

Parr and colleagues [10] reported that high Notch2 mRNA expression levels were associated with good clinical outcomes, thus suggesting a tumor suppressor role for Notch2 in breast cancer. This hypothesis was supported by O'Neill and colleagues [29], who showed that ectopic expression of active N2ICD in breast cancer MDA-MB-231 cells retarded cell growth and induced apoptosis in vitro whereas ectopic expression of N4ICD enhanced cellular proliferation. However, the tumor suppressor activity of Notch2 has been reported only in MDA-MB-231, a basal-like cell line. Whether it also holds true in a luminal type or HER-2 type of breast cancers (or both) remains to be investigated. Another group has suggested a positive correlation between Notch 2 and HER-2 expression levels [30]. In addition, a positive association between Notch2 expression and a single-nucleotide polymorphism associated with high risk of breast cancer has been reported in a subset of patients with breast cancer (ER-positive with wild-type p53) [31]. These two latter observations suggest that Notch2 might function as an oncogene rather than as a tumor suppressor. It is interesting to note that, while transgenic mice expressing active Notch1/3/4 intracellular domains have been generated and all develop mammary carcinoma, no transgenic mouse constitutively expressing active N2ICD specifically in mammary epithelium has been reported.

\section{Dependence of breast cancer on Notch signaling}

Whether Notch inhibition can be exploited to treat breast cancer depends on whether breast cancer cells require Notch signaling for their survival or malignant phenotype or both. The most widely used approach to block Notch signaling in breast cancer cells, as in cells of other types of cancer, has been the use of GSIs. Several early studies reported that the GSI z-Leu-Leu-Nle-CHO, commonly referred as GSI I, could induce cell cycle arrest and apoptosis in breast cancer cells and that ER-negative cell lines are more sensitive $[12,32,33]$. However, we have found that the cytotoxicity of GSI I is mediated by proteasome inhibition rather than $\gamma$-secretase inhibition [27]. In addition, we found that more specific GSIs DAPT or L-685,458 - had no significant cytotoxicity in six breast cancer cell lines with different genetic backgrounds. The lack of cytotoxicity of DAPT in breast cancer cells was confirmed by a recent study [28]. While this is discouraging, the recent study by Dong and colleagues [28] demonstrated that, although treatment with DAPT or an epidermal growth factor receptor
(EGFR) inhibitor, gefitinib, alone showed no cytotoxicity, the combination of DAPT with gefitinib induced significant growth arrest and cell death in basal-like breast cancer cells. The authors did not determine whether this combination is also effective in luminal and HER-2 type breast cancer cells.

It is important to recognize that, while these results suggest a clinical potential for Notch inhibition in combination therapy, there are more than $20 \gamma$-secretase substrates and many have biological functions in breast cancer [34]. Therefore, it would be useful to block Notch signaling by means of a more specific approach. For example, dominant negative MAML1 (dnMAML1) could be used in breast cancer cell lines in order to confirm that Notch signaling was responsible for the observed GSI cytotoxicity. To this end, Meurette and colleagues [35] reported that DAPT sensitized ER-positive MCF-7 and basal-like DCIS.com cells to a DNA-damaging drug (melphalan), although DAPT alone was not cytotoxic. In that study, transient expression of Numb or dnMAML1 was also used, with similar effects, to downregulate Notch activity. Since Notch inhibition has also been reported to sensitize breast cancer cells to tamoxifen and trastuzumab $[12,24]$, Notch inhibition in combination with other drugs holds a promising future in breast cancer management. Its potential as a monotherapy is more doubtful.

In contrast to reports that GSI treatment alone did not induce significant cytotoxicity in breast cancer cells, several studies reported that knocking down individual Notch paralogs by short interfering RNA (siRNA) was cytotoxic. Yamaguchi and colleagues [26] found that, although Notch1 knockdown had only a slight effect, Notch3 knockdown significantly impaired the growth of HER-2/neu-negative, but not -positive, cells. However, others have shown that knockdown of Notch1 by siRNA inhibits breast cancer cell growth $[12,24,36]$. Since the specificity of siRNA has always been a concern in knockdown experiments, it would be more convincing if the cytotoxicity could be reversed by expression of siRNAresistant full-length Notch receptors. It is critical, however, that full-length rather than simply the NICD constructs be used. It has been reported that N1ICD showed a dose-dependent effect on the phenotypic response in mammary epithelial MCF-10A cells [37], and overexpression of N1ICD has been shown to protect a number of cancer cell lines from several chemotherapy drugs [38]. Unlike full-length Notch proteins, NICD fragments will constitutively activate the Notch pathway and are likely to accumulate in vast excess of what would be generated by cleavage of endogenous Notch or a transfected full-length Notch construct. Therefore, the phenotype reversion arising from expression of NICD could be the result of overexpression rather than restoration of NICD. 
Alternatively, the efficacy of blocking the signaling of individual Notch paralogs could be tested using wellcharacterized, paralog-specific antagonizing antibodies that have recently been developed $[39,40]$. If downregulating the activity of individual Notch paralogs indeed induces cell growth arrest or cell death (despite the lack of significant cytotoxicity associated with treatment with GSI alone), this would suggest that other $\gamma$-secretase substrates function antagonistically to the pro-survival Notch signaling. Determining whether this is the case is essential to the development of effective therapeutic approaches to block oncogenic Notch signaling in the treatment of breast cancer.

\section{Notch and breast cancer stem cells}

Another motivation for exploring Notch inhibition for breast cancer treatment is that Notch signaling has been proposed to maintain the stemness of breast cancer stem cells (CSCs). Farnie and colleagues [41] examined the factors that affect the ability of breast cancer cells to form mammospheres, an indicator of stemness of breast CSCs. The authors found that DAPT treatment reduced the mammosphere-forming efficiency (MFE) of primary breast cancer cells. Furthermore, treatment with a polyclonal Notch4 neutralizing antibody reduced MFE to a greater extent than did DAPT treatment, suggesting that Notch4 signaling was involved in maintaining breast CSC stemness. Consistent with this, Harrison and colleagues [42] reported that Notch4, but not Notch1, signaling is important for regulating breast CSC activity. N1ICD levels were lower and N4ICD levels were higher in the CSC subpopulation compared with the non-CSC subpopulations. In addition, Notch4 knockdown produced a greater reduction in the MFE and tumorigenic potential in xenografts than did Notch1 knockdown in MCF-7 cells. However, the antibodies used do not specifically recognize the active NICD; more importantly, treatment with GSIs reduced the signal of 'N1ICD', but not that of 'N4ICD', as determined by immunoblotting in both MCF-7 and MDA-MB-231 cells. Since cleavage of int3/ Notch4 has been previously reported to be sensitive to GSI treatment $[43,44]$, this raises concerns over the specificity of the anti-Notch 4 antibody and, consequently, the validity of these observations. Finally, it should be noted that DAPT treatment reduced the MFE by only $20 \%$. If blocking Notch4 signaling is more effective in abrogating the stemness of breast CSCs than DAPT treatment, then some unidentified $\gamma$-secretase substrates must antagonize Notch4 activity.

\section{Targeting RBPjk-independent Notch signaling}

In addition to the CSL-dependent canonical Notch signaling described above, CSL-independent Notch activities have been reported, although most of the underlying molecular mechanisms are not well understood (reviewed in [45]). One of the best-characterized mechanisms is N1ICD inhibition of myogenesis by its competition with MEF2C for MAML1, the transcriptional activator required for myogenesis [46]. The other possible mechanisms underlying CSL-independent Notch activities include the formation of novel NICDcontaining protein complexes. It has been reported that NICD could associate with NFאB, IKK $\alpha$, Smad3, HIF-1 $\alpha$, YY1, and JNK, although the conditions for these associations have not been well characterized $[45,47,48]$.

The relative contributions of CSL-dependent canonical and CSL-independent non-canonical Notch activities to Notch-induced tumorigenesis have not been extensively investigated, although early studies suggested that noncanonical Notch activities might be involved [49]. A compelling study by Raafat and colleagues [50] demonstrated that active N4ICD can induce mammary gland carcinoma in the absence of RBPjk, the murine ortholog of the sequence-specific DNA-binding protein CSL. What mediates RBPjk-independent oncogenic Notch4 signaling remains to be determined, as does whether the RBPjk-independent tumorigenic activity described for Notch4 is a property that applies to other Notch paralogs. Nonetheless, this unexpected observation raises an intriguing question: can Notch-dependent cancer cells be killed by targeting RBPjк-independent Notch signaling?

When we expressed different levels of Flag-tagged N1ICD in MCF-7 cells, we found by co-immunoprecipitation that the amount of canonical N1ICD/RBPjk/ MAML protein complex became proportionally less with increasing levels of N1ICD [51]. Further investigation by examining the molecular weight distributions of RBPjk and three MAML homologs while increasing the expression of Flag-N1ICD revealed that MAML proteins are the limiting factor for continuous formation of canonical NICD/RBPjk/MAML protein complex. As the N1ICD expression increased, the co-elution of N1ICD and MAML decreased. On the basis of this, we propose that active NICD could function through three mechanisms: (a) activation of direct Notch target genes by the NICD/RBPjk/MAML transactivation complex, (b) competing MAML (or RBPjк away from other interacting proteins, thus inhibiting their activities), and (c) introducing new signaling pathways through the formation of novel NICD complexes (Figure 1). The third mechanism may be responsible for oncogenic Notch4 signaling in the absence of RBPjk but all Notch paralogs are expected to have this potential. It is possible that the activation of novel signaling pathways may arise only after NICD saturates MAML proteins. This is more likely to occur in cancer cells, in which Notch is aberrantly activated, as opposed to normal cells, in which Notch activation is tightly regulated. If this hypothesis is correct, 


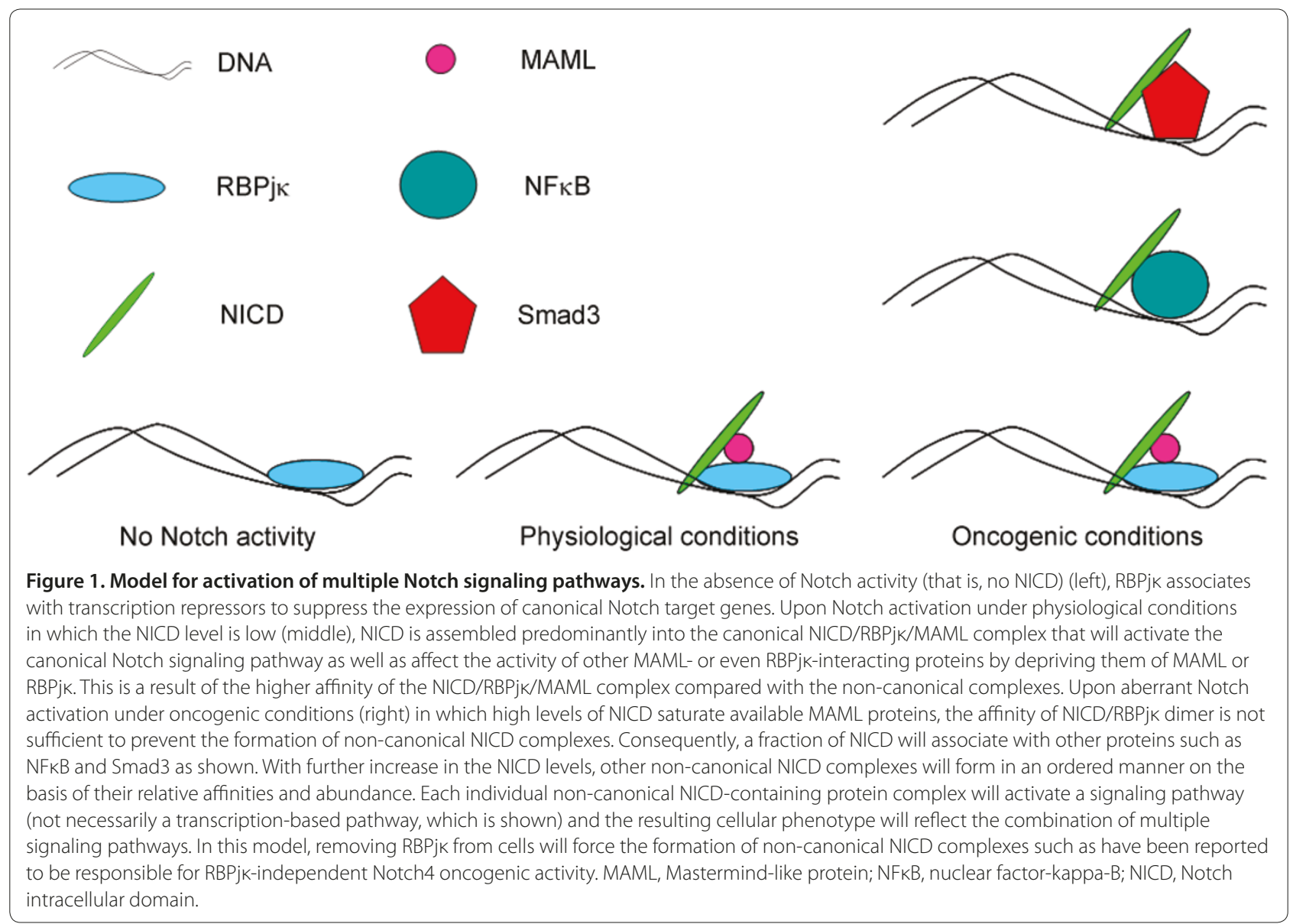

targeting RBPjk-independent Notch signaling might be a safe but effective approach to block oncogenic Notch activity while preserving the physiological activities of Notch which may be important for normal tissue functioning.

There are several indirect lines of evidence that support our hypothesis: (a) the dose-dependent nature of the transformation potential of activating Notch1 mutant alleles in T-ALL might reflect the fact that the N1ICD level needs to reach a threshold above which N1ICD could activate several non-canonical Notch signaling pathways by forming novel NICD protein complexes; (b) NICDs have been reported to associate with NFKB, IKK $\alpha$, Smad3, HIF1 $\alpha, \mathrm{YY} 1$, and JNK, all of which have been implicated in oncogenesis and cancer cell survival $[45,47,48]$; and (c) inhibition of NFKB activity delayed the onset of N1ICD-induced oncogenesis of T-ALL [52]. Therefore, a better understanding of the components of RBPjk-independent oncogenic Notch signaling pathways and their contribution to Notch-induced tumorigenesis would help us to develop new strategies to treat Notchdependent cancers.

\section{Conclusions}

Notch signaling is attractive as a potential therapeutic target for breast cancer management. However, after years of intensive investigation, there are still many uncertainties that prevent the successful exploitation of this target. To a large degree, these discrepancies arise from the complex nature of the Notch signaling pathway as well from limitations of existing reagents. We believe that it is essential to develop well-characterized and highly specific antibodies that can be used to directly and quantitatively detect the activation status of individual Notch paralogs in different types of breast cancers. In addition, since there are several approaches - specific GSIs, dnMAML1, and inhibitory antibodies - available to block Notch signaling, it is important to employ more than one approach when investigating the biological outcomes of Notch inhibition. More emphasis should also be placed on specificity rather than efficacy if we are to improve upon the current therapeutic use of GSIs. Finally, it will be important to investigate the contributions of non-canonical Notch activities to the oncogenesis and cell survival of Notch-dependent breast 
cancers. A better understanding of non-canonical Notch activities may lead to development of therapeutic strategies that minimize harm in normal tissue while effectively inhibiting the oncogenic Notch signaling.

\section{Abbreviations}

CSC, cancer stem cell; CSL, CBF-1/Su(H)/Lag-1; DCIS, ductal carcinoma in situ; dnMAML1, dominant negative Mastermind-like protein 1; ER, estrogen receptor; GSI, Y-secretase inhibitor; GSI I, Y-secretase inhibitor z-Leu-LeuNle-CHO; HUT, hyperplasia of usual type; IDC, infiltrating ductal carcinoma; ILC, infiltrating lobular carcinoma; MAML, Mastermind-like protein; MFE, mammosphere-forming efficiency; MMTV, mouse mammary tumor virus; N1/2/3/4ICD, Notch1/2/3/4 intracellular domain; NICD, Notch intracellular domain; NRR, negative regulatory region; PEST, proline/glutamic acid/serine/ threonine-rich; PR, progesterone receptor; siRNA, short interfering RNA; T-ALL, T-cell acute lymphoblastic leukemia.

\section{Competing interests}

The authors declare that they have no competing interests.

\section{Acknowledgments}

This project has been made possible through a grant from the Alberta Cancer Research Institute (ACRI) and the Alberta Cancer Foundation. JH is supported by an ACRI Graduate Studentship and the Canadian Institutes of Health Research (CIHR) Translation Research Training in Cancer program. MJH is an Alberta Heritage Foundation for Medical Research Senior Scholar.

Published: 31 May 2011

\section{References}

1. Kopan R, llagan MX: The canonical Notch signaling pathway: unfolding the activation mechanism. Cell 2009, 137:216-233.

2. Gallahan D, Callahan R: The mouse mammary tumor associated gene INT3 is a unique member of the NOTCH gene family (NOTCH4). Oncogene 1997 14:1883-1890

3. Gallahan D, Jhappan C, Robinson G, Hennighausen L, Sharp R, Kordon E, Callahan R, Merlino G, Smith GH: Expression of a truncated Int3 gene in developing secretory mammary epithelium specifically retards lobular differentiation resulting in tumorigenesis. Cancer Res 1996, 56:1775-1785.

4. Hu C, Dievart A, Lupien M, Calvo E, Tremblay G, Jolicoeur P: Overexpression of activated murine Notch 1 and Notch3 in transgenic mice blocks mammary gland development and induces mammary tumors. Am J Pathol 2006, 168:973-990.

5. Reedijk M, Odorcic S, Chang L, Zhang H, Miller N, McCready DR, Lockwood G, Egan SE: High-level coexpression of JAG1 and NOTCH1 is observed in human breast cancer and is associated with poor overall survival. Cancer Res 2005, 65:8530-8537.

6. Rizzo P, Osipo C, Foreman K, Golde T, Osborne B, Miele L: Rational targeting of Notch signaling in cancer. Oncogene 2008, 27:5124-5131.

7. Shi W, Harris AL: Notch signaling in breast cancer and tumor angiogenesis: cross-talk and therapeutic potentials. J Mammary Gland Biol Neoplasia 2006, 11:41-52.

8. Weng AP, Ferrando AA, Lee W, Morris JPt, Silverman LB, Sanchez-Irizarry C, Blacklow SC, Look AT, Aster JC: Activating mutations of NOTCH1 in human T cell acute lymphoblastic leukemia. Science 2004, 306:269-271.

9. Lee $\mathrm{SH}$, Jeong EG, Yoo NJ, Lee SH: Mutational analysis of NOTCH1, 2, 3 and 4 genes in common solid cancers and acute leukemias. Apmis 2007, 115:1357-1363.

10. Parr C, Watkins G, Jiang WG: The possible correlation of Notch-1 and Notch-2 with clinical outcome and tumour clinicopathological parameters in human breast cancer. Int J Mol Med 2004, 14:779-786.

11. Zardawi SJ, Zardawi I, McNeil CM, Millar EK, McLeod D, Morey AL, Crea P, Murphy NC, Pinese M, Lopez-Knowles E, Oakes SR, Ormandy CJ, Qiu MR, Hamilton A, Spillane A, Soon Lee C, Sutherland RL, Musgrove EA, O'Toole SA: High Notch1 protein expression is an early event in breast cancer development and is associated with the HER-2 molecular subtype. Histopathology 2010, 56:286-296.

12. Rizzo P, Miao H, D'Souza G, Osipo C, Song LL, Yun J, Zhao H, Mascarenhas J, Wyatt D, Antico G, Hao L, Yao K, Rajan P, Hicks C, Siziopikou K, Selvaggi S, Bashir A, Bhandari D, Marchese A, Lendahl U, Qin JZ, Tonetti DA, Albain K,
Nickoloff BJ, Miele L: Cross-talk between notch and the estrogen receptor in breast cancer suggests novel therapeutic approaches. Cancer Res 2008, 68:5226-5235.

13. Mittal S, Subramanyam D, Dey D, Kumar RV, Rangarajan A: Cooperation of Notch and Ras/MAPK signaling pathways in human breast carcinogenesis. Mol Cancer 2009, 8:128.

14. Li Y, Burns JA, Cheney CA, Zhang N, Vitelli S, Wang F, Bett A, Chastain M, Audoly LP, Zhang ZQ: Distinct expression profiles of Notch-1 protein in human solid tumors: Implications for development of targeted therapeutic monoclonal antibodies. Biologics 2010, 4:163-171.

15. McGill MA, Dho SE, Weinmaster G, McGlade CJ: Numb regulates postendocytic trafficking and degradation of Notch1.J Bio/ Chem 2009 284:26427-26438.

16. Pece S, Serresi M, Santolini E, Capra M, Hulleman E, Galimberti V, Zurrida S, Maisonneuve P, Viale G, Di Fiore PP: Loss of negative regulation by Numb over Notch is relevant to human breast carcinogenesis. J Cell Biol 2004, 167:215-221.

17. Stylianou S, Clarke RB, Brennan K: Aberrant activation of notch signaling in human breast cancer. Cancer Res 2006, 66:1517-1525.

18. Hartman J, Lam EW, Gustafsson JA, Strom A: Hes-6, an inhibitor of Hes-1, is regulated by 17 beta-estradiol and promotes breast cancer cell proliferation. Breast Cancer Res 2009, 11:R79.

19. Chiang MY, Xu L, Shestova O, Histen G, L'Heureux S, Romany C, Childs ME, Gimotty PA, Aster JC, Pear WS: Leukemia-associated NOTCH1 alleles are weak tumor initiators but accelerate K-ras-initiated leukemia. J Clin Invest 2008, 118:3181-3194.

20. Reedijk M, Pinnaduwage D, Dickson BC, Mulligan AM, Zhang H, Bull SB, O'Malley FP, Egan SE, Andrulis IL: JAG1 expression is associated with a basal phenotype and recurrence in lymph node-negative breast cancer. Breast Cancer Res Treat 2008, 111:439-448.

21. Sethi N, Dai X, Winter CG, Kang Y: Tumor-derived JAGGED1 promotes osteolytic bone metastasis of breast cancer by engaging notch signaling in bone cells. Cancer Cell 2011, 19:192-205.

22. Sørlie T, Perou CM, Tibshirani R, Aas T, Geisler S, Johnsen H, Hastie T, Eisen MB, van de Rijn M, Jeffrey SS, Thorsen T, Quist H, Matese JC, Brown PO, Botstein D, Eystein Lønning P, Børresen-Dale AL: Gene expression patterns of breast carcinomas distinguish tumor subclasses with clinical implications. Proc Natl Acad Sci U S A 2001, 98:10869-10874.

23. Dickson BC, Mulligan AM, Zhang H, Lockwood G, O'Malley FP, Egan SE, Reedijk M: High-level JAG1 mRNA and protein predict poor outcome in breast cancer. Mod Pathol 2007, 20:685-693.

24. Osipo C, Patel P, Rizzo P, Clementz AG, Hao L, Golde TE, Miele L: ErbB-2 inhibition activates Notch-1 and sensitizes breast cancer cells to a gamma-secretase inhibitor. Oncogene 2008, 27:5019-5032.

25. Cohen B, Shimizu M, Izrailit J, Ng NF, Buchman Y, Pan JG, Dering J, Reedijk M: Cyclin D1 is a direct target of JAG1-mediated Notch signaling in breast cancer. Breast Cancer Res Treat 2010, 123:113-124.

26. Yamaguchi N, Oyama T, Ito E, Satoh H, Azuma S, Hayashi M, Shimizu K, Honma R, Yanagisawa Y, Nishikawa A, Kawamura M, Imai J, Ohwada S, Tatsuta K, Inoue J, Semba K, Watanabe S: NOTCH3 signaling pathway plays crucial roles in the proliferation of ErbB2-negative human breast cancer cells. Cancer Res 2008, 68:1881-1888.

27. Han J, Ma I, Hendzel MJ, Allalunis-Turner J: The cytotoxicity of gammasecretase inhibitor I to breast cancer cells is mediated by proteasome inhibition, not by gamma-secretase inhibition. Breast Cancer Res 2009, 11:R57.

28. Dong Y, Li A, Wang J, Weber JD, Michel LS: Synthetic lethality through combined Notch-epidermal growth factor receptor pathway inhibition in basal-like breast cancer. Cancer Res 2010, 70:5465-5474

29. O'Neill CF, Urs S, Cinelli C, Lincoln A, Nadeau RJ, Leon R, Toher J, Mouta-Bellum C, Friesel RE, Liaw L: Notch2 signaling induces apoptosis and inhibits human MDA-MB-231 xenograft growth. Am J Patho/ 2007, 171:1023-1036.

30. Florena AM, Tripodo C, Guarnotta C, Ingrao S, Porcasi R, Martorana A, Lo Bosco G, Cabibi D, Franco V: Associations between Notch-2, Akt-1 and HER2/neu expression in invasive human breast cancer: a tissue microarray immunophenotypic analysis on 98 patients. Pathobiology 2007, 74:317-322

31. Fuwa TJ, Hori K, Sasamura T, Higgs J, Baron M, Matsuno K: The first deltex null mutant indicates tissue-specific deltex-dependent Notch signaling in Drosophila. Mol Genet Genomics 2006, 275:251-263.

32. Lee CW, Raskett CM, Prudovsky I, Altieri DC: Molecular dependence of estrogen receptor-negative breast cancer on a notch-survivin signaling 
axis. Cancer Res 2008, 68:5273-5281.

33. Rasul S, Balasubramanian R, Filipovic A, Slade MJ, Yague E, Coombes RC: Inhibition of gamma-secretase induces $\mathrm{G} 2 / \mathrm{M}$ arrest and triggers apoptosis in breast cancer cells. Br J Cancer 2009, 100:1879-1888.

34. Lleo A: Activity of gamma-secretase on substrates other than APP. Curr Top Med Chem 2008, 8:9-16.

35. Meurette O, Stylianou S, Rock R, Collu GM, Gilmore AP, Brennan K: Notch activation induces Akt signaling via an autocrine loop to prevent apoptosis in breast epithelial cells. Cancer Res 2009, 69:5015-5022.

36. Zang S, Chen F, Dai J, Guo D, Tse W, Qu X, Ma D, Ji C: RNAi-mediated knockdown of Notch-1 leads to cell growth inhibition and enhanced chemosensitivity in human breast cancer. Oncol Rep 2010, 23:893-899.

37. Mazzone M, Selfors LM, Albeck J, Overholtzer M, Sale S, Carroll DL, Pandya D, Lu Y, Mills GB, Aster JC, Artavanis-Tsakonas S, Brugge JS: Dose-dependent induction of distinct phenotypic responses to Notch pathway activation in mammary epithelial cells. Proc Natl Acad Sci U S A 2010, 107:5012-5017.

38. Mungamuri SK, Yang $X$, Thor AD, Somasundaram K: Survival signaling by Notch1: mammalian target of rapamycin (mTOR)-dependent inhibition of p53. Cancer Res 2006, 66:4715-4724

39. Li K, Li Y, Wu W, Gordon WR, Chang DW, Lu M, Scoggin S, Fu T, Vien L, Histen G, Zheng J, Martin-Hollister R, Duensing T, Singh S, Blacklow SC, Yao Z, Aster JC, Zhou BB: Modulation of Notch signaling by antibodies specific for the extracellular negative regulatory region of NOTCH3. J Biol Chem 2008, 283:8046-8054

40. Wu Y, Cain-Hom C, Choy L, Hagenbeek TJ, de Leon GP, Chen Y, Finkle D, Venook R, Wu X, Ridgway J, Schahin-Reed D, Dow GJ, Shelton A, Stawicki S, Watts RJ, Zhang J, Choy R, Howard P, Kadyk L, Yan M, Zha J, Callahan CA, Hymowitz SG, Siebel CW: Therapeutic antibody targeting of individual Notch receptors. Nature 2010, 464:1052-1057.

41. Farnie G, Clarke RB, Spence K, Pinnock N, Brennan K, Anderson NG, Bundred $\mathrm{NJ}$ : Novel cell culture technique for primary ductal carcinoma in situ: role of Notch and epidermal growth factor receptor signaling pathways. J Nat Cancer Inst 2007, 99:616-627.

42. Harrison H, Farnie G, Howell SJ, Rock RE, Stylianou S, Brennan KR, Bundred NJ, Clarke RB: Regulation of breast cancer stem cell activity by signaling through the Notch4 receptor. Cancer Res 2010, 70:709-718.

43. Das I, Craig C, Funahashi Y, Jung KM, Kim TW, Byers R, Weng AP, Kutok JL, Aster JC, Kitajewski J: Notch oncoproteins depend on gamma-secretase/ presenilin activity for processing and function. J Biol Chem 2004, 279:30771-30780.
44. Saxena MT, Schroeter EH, Mumm JS, Kopan R: Murine notch homologs (N1-4) undergo presenilin-dependent proteolysis. J Bio/ Chem 2001, 276:40268-40273.

45. Heitzler P: Biodiversity and noncanonical Notch signaling. Curr Top Dev Biol 2010, 92:457-481.

46. Shen H, McElhinny AS, Cao Y, Gao P, Liu J, Bronson R, Griffin JD, Wu L: The Notch coactivator, MAML1, functions as a novel coactivator for MEF2Cmediated transcription and is required for normal myogenesis. Genes Dev 2006, 20:675-688

47. Liao WR, Hsieh RH, Hsu KW, Wu MZ, Tseng MJ, Mai RT, Wu Lee YH, Yeh TS: The CBF1-independent Notch1 signal pathway activates human c-myc expression partially via transcription factor YY1. Carcinogenesis 2007 28:1867-1876.

48. Kim JW, Kim MJ, Kim KJ, Yun HJ, Chae JS, Hwang SG, Chang TS, Park HS, Lee KW, Han PL, Cho SG, Kim TW, Choi EJ: Notch interferes with the scaffold function of JNK-interacting protein 1 to inhibit the JNK signaling pathway. Proc Natl Acad Sci U S A 2005, 102:14308-14313.

49. Dumont E, Fuchs KP, Bommer G, Christoph B, Kremmer E, Kempkes B: Neoplastic transformation by Notch is independent of transcriptional activation by RBP-J signalling. Oncogene 2000, 19:556-561.

50. Raafat A, Lawson S, Bargo S, Klauzinska M, Strizzi L, Goldhar AS, Buono K, Salomon D, Vonderhaar BK, Callahan R: Rbpj conditional knockout reveals distinct functions of Notch4/Int3 in mammary gland development and tumorigenesis. Oncogene 2009, 28:219-230.

51. Han J, Allalunis-Turner J, Hendzel MJ: Characterization and comparison of protein complexes initiated by the intracellular domain of individual Notch paralogs. Biochem Biophys Res Commun 2011, 407:479-485.

52. Vilimas T, Mascarenhas J, Palomero T, Mandal M, Buonamici S, Meng F, Thompson B, Spaulding C, Macaroun S, Alegre ML, Kee BL, Ferrando A, Miele L, Aifantis I: Targeting the NF-kappaB signaling pathway in Notch1induced T-cell leukemia. Nat Med 2007, 13:70-77.

doi:10.1186/bcr2875

Cite this article as: Han J, et al.: Notch signaling as a therapeutic target for breast cancer treatment? Breast Cancer Research 2011, 13:210. 